\title{
Horloges à réseau optique au strontium
}

\section{Strontium optical lattice clocks}

\author{
Jérôme LODEWYCK, Rodolphe LE TARGAT, Sławomir BILICKI, Eva BOOKJANS et Grégoire VALLET \\ LNE-SYRTE, Observatoire de Paris, PSL Research University, CNRS, Sorbonne Universités, UPMC Univ. Paris 06, 61 Avenue de l'Observatoire, \\ 75014 Paris, France, jerome.lodewyck@obspm.fr.
}

\begin{abstract}
Résumé
Dans les horloges à réseau optique, des atomes froids confinés dans un piège dipolaire en forme de réseau sont interrogés par un laser ultrastable. Elles sont devenues les meilleurs étalons de fréquence car elles allient à la fois une résonance étroite dans le domaine optique, et donc grand facteur de qualité, et un grand nombre d'atomes interrogés simultanément. Dans cet article, nous présentons les derniers développements de ces horloges en termes de stabilité de fréquence et d'exactitude, en décrivant les résultats obtenus avec deux horloges à réseau optique au strontium développées au LNE-SYRTE.
\end{abstract}

MOTS CLÉS : MÉTROLOGIE DU TEMPS ET DES FRÉQUENCES, HORLOGES À RÉSEAU OPTIQUE, ATOMES FROIDS, LASERS ULTRASTABLES.

\begin{abstract}
In optical lattice clocks, an ensemble of cold neutral atoms confined in a lattice-shaped dipole trap are probed by an ultrastable laser. They have recently become the best frequency references, because they combine a narrow atomic resonance in the optical domain - hence a large quality factor - and a large number of simultaneously probed atoms. In this paper, we present the latest developments of these clocks, both in terms of frequency stability and accuracy, by focusing on two strontium optical lattice clocks under operation at LNE-SYRTE.
\end{abstract}

KEY WORDS: TIME AND FREQUENCY METROLOGY, OPTICAL LATTICE CLOCKS, COLD ATOMS, ULTRASTABLE LASERS.

\section{Introduction}

Les horloges atomiques sont fondées sur l'asservissement de la fréquence d'un oscillateur local sur une transition atomique étroite via l'interaction entre les atomes et une onde électromagnétique asservie sur l'oscillateur local. Les horloges au césium utilisent pour cela une transition hyperfine de l'état fondamental, située dans le domaine micro-onde du spectre électro-magnétique. Ces horloges, actuellement au stade opérationnel, sont utilisées couramment pour calibrer les échelles de temps internationales (le temps universel coordonné UTC et le temps atomique international TAI) via des comparaisons satellitaires, ainsi que pour réaliser la définition de la seconde du Système international d'unités (SI). L'exactitude de ces étalons primaires de fréquence, au niveau de quelques $10^{-16}[1,2]$, résulte notamment de l'utilisation d'atomes ultra-froids en chute libre pour lesquels le déplacement de fréquence Doppler dû au mouvement des atomes est fortement réduit et le temps d'interrogation de la transition d'horloge est de l'ordre de la seconde.

Malgré ce succès, les horloges atomiques micro-onde arrivent à leurs performances ultimes. Pour réaliser de meilleurs étalons de fréquences, des horloges utilisant des transitions atomiques étroites dans le domaine optique, et donc avec un facteur de qualité $10^{4}$ fois plus élevé, sont développées. Pour s'affranchir de l'effet Doppler, les espèces atomiques utilisées dans ces horloges doivent être confinées et refroidies dans un piège électro-magnétique.

Pour cela, deux pistes sont poursuivies : d'une part les horloges piégeant un ion unique dans un champ électromagnétique radiofréquence, et d'autre part les horloges à réseau optique piégeant des atomes neutres dans une onde laser stationnaire par la force dipolaire [3]. Ces dernières, de par le meilleur rapport signal à bruit résultant du grand nombre d'atomes qu'on peut interroger simultanément, sont récemment devenues les meilleures horloges atomiques, aussi bien en termes de résolution statistique sur la fréquence (ou stabilité de fréquence) au niveau de $2 \times 10^{-16} / \sqrt{\tau}$ où $\tau$ est le temps d'intégration exprimé en secondes [4-7], que sur le contrôle des effets systématiques perturbateurs (ou exactitude) au niveau de $2 \times 10^{-18}[6,8]$. 
Le LNE-SYRTE développe depuis 2000 des étalons de fréquence optiques avec des atomes neutres. Deux horloges à réseau optique ( $\mathrm{Sr} 1$ et $\mathrm{Sr} 2)$ utilisant des atomes de strontium sont maintenant en fonctionnement. La stabilité de fréquence de ces horloges est de $10^{-15} / \sqrt{\tau}$, et l'exactitude de $\mathrm{Sr} 2$ est de $4 \times 10^{-17}$. Les horloges à réseau optique, bien que présentant déjà des performances exceptionnelles, ont encore des perspectives d'amélioration, notamment grâce aux progrès des sources laser ultrastables qui laissent entrevoir une résolution statistique de l'ordre de $10^{-17}$ après $1 \mathrm{~s}$ d'intégration.

Dans cet article, nous expliquerons brièvement le fonctionnement des horloges à réseau optique (paragraphe 2), puis nous détaillerons les limites actuelles et les perspectives concernant leur stabilité (paragraphe 3) et leur exactitude (paragraphe 4). Pour tirer parti des performances des horloges atomiques, il est nécessaire de les comparer entre elles, aussi bien localement au LNESYRTE, que de façon distante via des moyens de transfert de fréquence (paragraphe 5). Ces comparaisons, en plus de démontrer la reproductibilité des horloges, offrent de nombreuses applications, notamment en géophysique ou en physique fondamentale. Dans la paragraphe 6 , nous ouvrirons la perspective que les horloges optiques, et en particulier les horloges à réseau optique au strontium, sont amenées à être utilisées pour redéfinir l'unité de temps du SI, la seconde.

\section{Principe de fonctionnement}

Le LNE-SYRTE a développé deux horloges à réseau optique au strontium dont le principe de fonctionnement est similaire. Certains aspects détaillés dans [9] ne sont que brièvement décrits ici.

Les horloges sont constituées de deux composants principaux. D'abord le système atomique proprement dit, constitué d'une enceinte à vide dans laquelle sont préparés les atomes froids de strontium piégés, entourée d'un système de lasers pour la capture et la manipulation des atomes. Ensuite, les atomes sont interrogés par un laser de très petite largeur de raie (inférieure à $1 \mathrm{~Hz}$ ), obtenu par la stabilisation d'une diode laser sur une cavité FabryPerot ultrastable. Ce laser ultrastable, commun aux deux horloges, est décrit dans la paragraphe 3.

Le refroidissement laser des atomes repose sur la structure atomique du strontium représentée sur la figure 1. Un jet de strontium collimaté issu d'un four chauffé à $860 \mathrm{~K}$ est ralenti par un ralentisseur Zeeman, puis capturé et refroidit à une température de $3 \mathrm{mK}$ dans un piège magnéto-optique (PMO), en utilisant la transition intense ${ }^{1} \mathrm{~S}_{0} \rightarrow{ }^{1} \mathrm{P}_{1}$ à la longueur d'onde de $461 \mathrm{~nm}$. Quelques $10^{6}$ atomes de ${ }^{87} \mathrm{Sr}$ sont ainsi capturés (fig. 2).

Le réseau optique est formé par une onde laser stationnaire à la longueur d'onde magique $\left(\lambda_{l}=813 \mathrm{~nm}\right.$, voir paragraphe 4.1) dans une cavité optique d'environ $4 \mathrm{~cm}$ de longueur placée dans l'enceinte à vide. En utilisant la méthode du drain atomique dans laquelle les atomes qui se trouvent au niveau du réseau optique sont

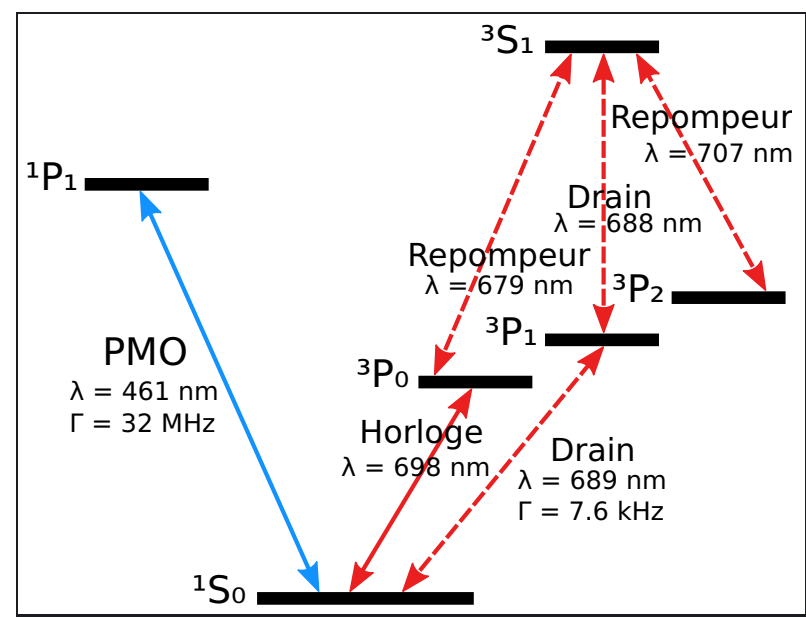

Fig. 1. - Niveaux d'énergie de l'atome de strontium. Le strontium possède deux électrons de valence, et donc des états singlets et des états triplets. Les résonances entre ces deux multiplicités sont très faiblement autorisées. Ainsi, la résonance ${ }^{1} \mathrm{~S}_{0} \rightarrow{ }^{3} \mathrm{P}_{1}$ permet le refroidissement sur raie étroite, et la largeur de raie de l'ordre du millihertz de la résonance ${ }^{1} \mathrm{~S}_{0} \rightarrow{ }^{3} \mathrm{P}_{0}$ est adaptée pour la transition d'horloge.

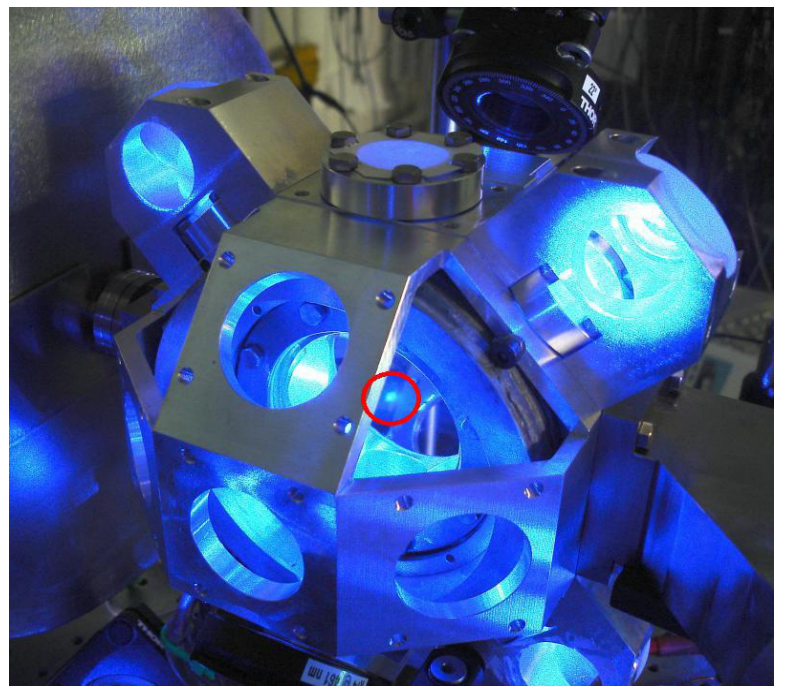

Fig. 2. - Zone de capture de l'horloge Sr2. Au centre, on aperçoit la fluorescence du piège magnéto-optique, désignée par une ellipse rouge.

pompés dans les états métastables ${ }^{3} \mathrm{P}_{0}$ et ${ }^{3} \mathrm{P}_{2}$ par deux faisceaux laser à $689 \mathrm{~nm}$ et $688 \mathrm{~nm}$ [9], on parvient à transférer environ $1 \%$ des atomes du PMO vers le réseau optique. La température de ces atomes est alors de l'ordre de grandeur de la profondeur du réseau optique, c'est-à-dire $200 \mu \mathrm{K}$, ou encore $1200 E_{R}$ ou $E_{R}=h^{2} / 2 m \lambda_{l}^{2}$ est l'énergie de recul associée à l'absorption d'un photon du réseau, et $m$ la masse d'un atome. Les atomes sont alors refroidis en utilisant la raie d'inter-combinaison ${ }^{1} \mathrm{~S}_{0} \rightarrow{ }^{3} \mathrm{P}_{1}$, soit par refroidissement Doppler dans les deux directions transverses au réseau jusqu'à une température de $10 \mu \mathrm{K}$, soit par refroidissement sur bandes latérales motionnelles dans la direction longitudinale jusqu'à une température de quelques microkelvins. 
Les horloges à réseau optique au strontium utilisent l'isotope fermionique ${ }^{87} \mathrm{Sr}$, car, du fait de son spin nucléaire non nul $F=9 / 2$, sa transition d'horloge ${ }^{1} \mathrm{~S}_{0} \rightarrow{ }^{3} \mathrm{P}_{0}$ n'est pas totalement interdite même avec un champ magnétique nul ou faible. Les isotopes bosoniques, malgré la plus grande abondance de deux d'entre eux, sont a contrario peu utilisés car l'excitation de leur résonance d'horloge nécessite une puissance optique et/ou un champ magnétique importants, dégradant ainsi le bilan d'exactitude. Le ${ }^{87} \mathrm{Sr}$ présente également un déplacement collisionnel réduit, grâce au principe d'exclusion de Pauli (paragraphe 4.2). La contrepartie est que chacun des niveaux d'horloge du ${ }^{87} \mathrm{Sr}$ possède 10 sousniveaux magnétiques $m_{F}$. Afin d'obtenir des résonances étroites et contrastées, il convient donc de pomper les atomes dans l'un de ces états (en pratique $m_{F}=9 / 2 \mathrm{ou}$ $m_{F}=-9 / 2$ ) avec un faisceau laser à $689 \mathrm{~nm}$ polarisé circulairement et aligné sur un champ magnétique statique d'environ $10^{-4} \mathrm{~T}$.

Les atomes sont ensuite interrogés avec le laser d'horloge à $698 \mathrm{~nm}$ pendant une durée de $200 \mathrm{~ms}$, limitée par le temps de cohérence de ce laser. Enfin, La probabilité de transition associée à cette interaction est mesurée en détectant à l'aide d'une caméra la fluorescence des atomes restés dans l'état fondamental soumis à un faisceau laser à $461 \mathrm{~nm}$. Le nombre d'atomes est normalisé par un pulse de repompage utilisant des lasers à $707 \mathrm{~nm}$ et $679 \mathrm{~nm}$ induisant le transfert de l'état ${ }^{3} \mathrm{P}_{0}$ vers l'état fondamental ${ }^{1} \mathrm{~S}_{0}$ suivi d'un deuxième pulse de détection.

Un intégrateur numérique est ensuite responsable d'asservir le laser ultrastable sur la transition d'horloge, en utilisant comme signal d'erreur la différence de probabilité de transition entre deux interrogations successives respectivement accordées sur chacun des flancs de la résonance.

\section{Stabilité de fréquence et limite ultime}

L'écart type d'Allan du bruit de fréquence du laser ultrastable asservi sur la transition d'horloge représente la stabilité de fréquence de l'horloge. La stabilité de fréquence de toutes les horloges atomiques est fondamentalement limitée par le bruit de projection quantique (BPQ), résultat de la projection aléatoire de la fonction d'onde dans l'état fondamental ou l'état excité de la transition d'horloge [10]. L'écart type d'Allan de cette limite s'écrit en fonction du facteur de qualité de la résonance $Q$, le nombre d'atomes $N$ et le temps de cycle $T_{c}$ :

$$
\sigma_{y}^{\mathrm{BPQ}}(\tau)=\frac{2}{\pi Q \sqrt{N}} \sqrt{\frac{T_{c}}{\tau}}
$$

La stabilité des meilleures horloges au césium et les horloges optiques à ion atteignent à l'heure actuelle le $\mathrm{BPQ}$, respectivement à quelques $10^{-14} / \sqrt{\tau}[1]$ et $2,8 \times$ $10^{-15} / \sqrt{\tau}[11]$. Les horloges à réseau optique, qui combinent à la fois un facteur de qualité élevé et un grand nombre d'atomes offrent un BPQ de l'ordre de $10^{-17} / \sqrt{\tau}$, qui n'est pas encore atteint.
La stabilité des horloges à réseau optique est en effet encore limitée par une source de bruit technique : l'effet Dick. Il s'agit d'un effet d'échantillonnage du bruit de fréquence du laser d'horloge par le cycle de l'horloge $[12,13]$. Dans la limite où le cycle d'horloge ne comprendrait pas de temps mort pour le chargement, la préparation et la détection des atomes, l'effet Dick serait nul et la stabilité des horloges à réseau optique atteindrait le BPQ [14].

Pour limiter l'effet Dick, deux pistes sont donc envisageables : la première est de réduire le bruit de fréquence du laser d'horloge à des fréquences de Fourier de $1 \mathrm{~Hz}$ à $100 \mathrm{~Hz}$ en concevant de nouvelles générations de lasers ultrastables. La deuxième est d'optimiser la séquence de l'horloge pour réduire au maximum le temps mort.

\subsection{Source laser ultrastable}

Le laser ultrastable utilisé au LNE-SYRTE pour les horloges au strontium est fondé sur la stabilisation d'une diode laser en cavité étendue sur une cavité Fabry-Perot horizontale de longueur $10 \mathrm{~cm}$ et de finesse 560000 . Les fluctuations relatives résiduelles de fréquence du laser asservi sont données par les fluctuations relatives de longueur de la cavité. Pour rendre l'effet des vibrations négligeables, la forme de la cavité a été optimisée par éléments finis [15]. Le bruit de fréquence du laser ultrastable est alors dû au bruit thermique des optiques de la cavité, et en particulier celui des traitements réfléchissants des miroirs [16] puisque les substrats des miroirs sont en silice fondue pour limiter leur bruit thermique. Les fluctuations de longueur dues aux fluctuations de température, particulièrement importantes à cause de l'utilisation de la silice fondue pour les substrats, sont rendues négligeables en entourant la cavité par trois boucliers thermiques, placés dans un double système d'enceintes à vide (fig. 3). La température de l'enceinte à vide intérieure est stabilisée, avec des fluctuations résiduelles de l'ordre de $1 \mathrm{mK}$, et les dérives de fréquence mesurées pour le laser asservi sur la cavité indiquent des fluctuations de température de la cavité inférieures à $1 \mathrm{nK}$ sur des échelles de temps inférieures à quelques minutes. Ces dérives de fréquence suivent un profil polynomial qui est annulé par un système de pré-compensation qui fait varier la fréquence d'un synthétiseur de fréquence.

Le bruit thermique de la cavité, mesuré avec les corrections de fréquence entre les horloges $\mathrm{Sr} 1$ et $\mathrm{Sr} 2$ et la fréquence du laser asservi sur la cavité ultrastable, est au niveau de $7 \times 10^{-16}$ sur des échelles de temps allant de quelques secondes à une heure, en accord avec la prédiction théorique. Avec ce niveau de bruit et un rapport cyclique de 0,25 entre le temps d'interrogation et le temps de cycle, l'effet Dick est mesuré à $10^{-15} / \sqrt{\tau}$ sur chacune des horloges $\mathrm{Sr} 1$ et $\mathrm{Sr} 2$ (fig. 4).

\subsection{Détection non destructive}

Un autre façon d'améliorer la stabilité de fréquence de l'horloge est d'augmenter le rapport cyclique, 


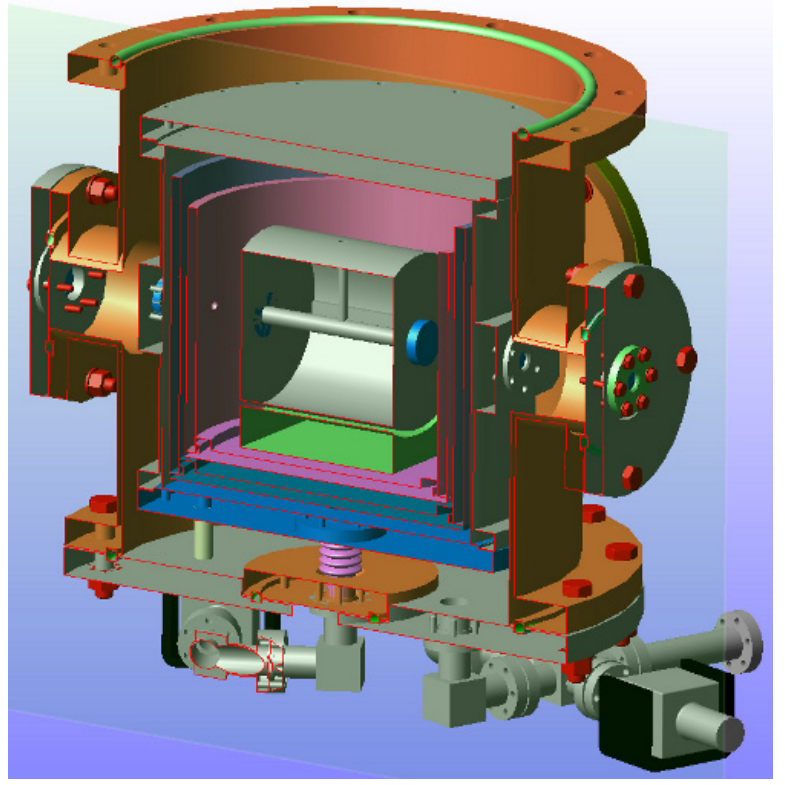

Fig. 3. - Vue en coupe des boucliers thermique et des deux enceintes à vide entourant la cavité ultrastable utilisée pour stabiliser la fréquence du laser d'horloge.

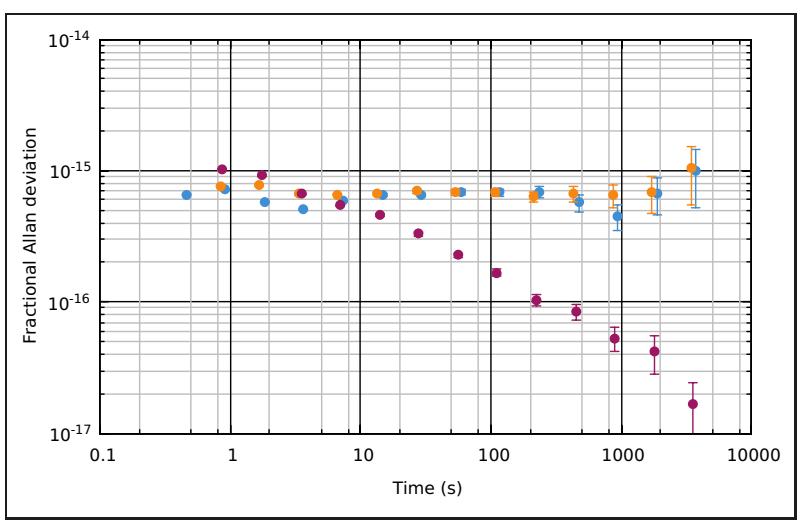

Fig. 4. - Stabilité de fréquence du laser d'horloge, comparée à l'horloge $\mathrm{Sr} 1$ (bleu) et $\mathrm{Sr} 2$ (orange). Une dérive polynomiale de la dérive de la cavité ultrastable est retiré. Le bruit de fréquence long terme du laser d'horloge s'élimine lorsque l'on compare les deux horloges (violet). La stabilité de cette comparaison reste toutefois limitée par l'échantillonnage par les atomes du bruit de fréquence du laser d'horloge situé aux fréquences multiples du cycle d'horloge (effet Dick).

c'est-à-dire diminuer le temps mort dédié à la capture et la préparation des atomes. À cette fin, nous avons démontré sur l'horloge $\mathrm{Sr} 1$ une détection non destructive de la probabilité de transition, qui remplace la détection par fluorescence, et qui permet de garder les atomes piégés dans le réseau optique. Cette détection permet donc d'éviter le rechargement d'atomes au cycle suivant, et ainsi de significativement améliorer le rapport cyclique. Cette détection tire parti du déphasage induit par les atomes sur un faisceau laser peu intense désaccordé par rapport à la transition ${ }^{1} \mathrm{~S}_{0} \rightarrow{ }^{1} \mathrm{P}_{1}$. Ce déphasage, mesuré par un interféromètre de Mach-Zehnder, est proportionnel au nombre d'atomes dans l'état fondamental ${ }^{1} \mathrm{~S}_{0}$ (fig. 5).

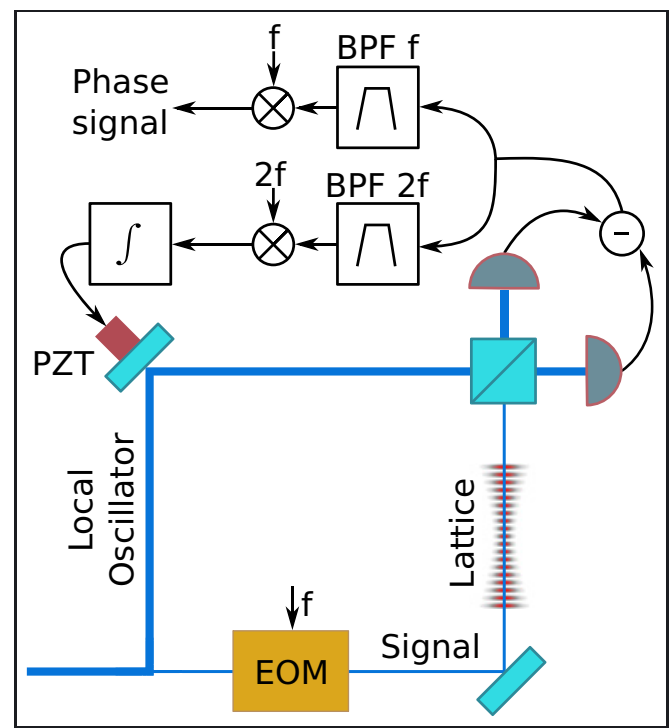

Fig. 5. - Schéma de la détection non destructive du nombre d'atomes dans l'état ${ }^{1} \mathrm{~S}_{0}$ fondée sur une mesure dispersive par un interféromètre de Mach-Zehnder. Le décalage de fréquence $f$ entre les deux bras de l'interféromètre, généré par un modulateur de phase électro-optique permet une détection hétérodyne limitée par le bruit de photon, par démodulation à la fréquence $f$. La démodulation à la fréquence $2 f$ permet un asservissement de la différence de marche physique entre les deux bras. Chaque signal est filtré avant démodulation par un filtre passe bande (BPF).

Nous avons démontré que le rapport signal à bruit de notre détection est fondamentalement limité par le bruit de photon du faisceau laser qui vient mesurer le nombre d'atomes. Ce rapport signal à bruit est de 100 pour $10^{4}$ atomes, c'est-à-dire au même niveau que le BPQ. Nous avons ensuite démontré que les atomes restent piégés dans le réseau optique après la détection, à condition d'élever la profondeur de piégeage aux alentours de $1000 E_{R}[17]$.

Une nouvelle version de cette détection est en cours de caractérisation. Elle tire parti d'une cavité optique entourant les atomes pour augmenter la longueur d'interaction entre le laser de détection et les atomes, améliorant ainsi le rapport signal à bruit. La robustesse de cette détection non destructive en cavité nous permettra de démontrer une stabilité améliorée sur des mesures d'horloge opérationnelles.

\section{4. Évaluation des effets systématiques}

Grâce à leur stabilité exceptionnelle, les horloges à réseau optique offrent une résolution statistique permettant la mesure et l'évaluation rapide des effets systématiques. Ainsi, une résolution de quelques $10^{-17}$ est obtenue après seulement quelques heures d'intégration sur les deux horloges du LNE-SYRTE (fig. 4).

Nous avons ainsi établi le bilan d'exactitude de l'horloge $\mathrm{Sr} 2$ au niveau de $4,1 \times 10^{-17}$, reproduit dans 
Tableau 1

Bilan d'exactitude de l'horloge $\mathrm{Sr} 2$ en valeur relative par rapport à la fréquence centrale $v=429,288 \mathrm{THz}$.

\begin{tabular}{|l|c|c|}
\hline Effet & Correction & Incertitude \\
\hline Déplacement lumineux du réseau & $-3 \times 10^{-17}$ & $2,5 \times 10^{-17}$ \\
Spectre du réseau & 0 & $1 \times 10^{-18}$ \\
Collisions atomes froids & 0 & $8 \times 10^{-18}$ \\
Charges statiques & 0 & $1,5 \times 10^{-18}$ \\
Dérive de phase dans les MAO & $-2 \times 10^{-18}$ & $2 \times 10^{-18}$ \\
Rayonnement du corps noir & $5,208 \times 10^{-15}$ & $1,8 \times 10^{-17}$ \\
Effet Zeeman quadratique & $1,317 \times 10^{-15}$ & $1,2 \times 10^{-17}$ \\
Distorsion de la résonance & 0 & $2 \times 10^{-17}$ \\
Rayonnement du corps noir four & 0 & $1 \times 10^{-17}$ \\
Erreur de l'intégrateur & 0 & $3 \times 10^{-18}$ \\
Collisions atomes chauds & 0 & $5 \times 10^{-18}$ \\
Déplacement lumineux de la sonde & $4 \times 10^{-19}$ & $4 \times 10^{-19}$ \\
\hline Total & $\mathbf{6 4 8 , 7 \times \mathbf { 1 0 } ^ { - 1 7 }}$ & $\mathbf{4 , 1 \times \mathbf { 1 0 } ^ { - 1 7 }}$ \\
\hline
\end{tabular}

le tableau 1. Les paragraphes ci-dessous détaillent l'évaluation des effets systématiques prédominants.

\subsection{Effets de piégeage}

L'effet systématique le plus caractéristique des horloges à réseau optique est le déplacement lumineux des niveaux d'horloge induit par l'interaction dipolaire entre les atomes et le laser de piégeage. Par exemple, à une profondeur modérée de $100 E_{R}$, ce déplacement est de l'ordre de $350 \mathrm{kHz}$, soit $8 \times 10^{-10}$ en valeur relative. La longueur d'onde magique pour les horloges à réseau optique est définie comme la longueur d'onde du laser de piégeage pour laquelle ce déplacement lumineux scalaire prédominant est identique pour les deux niveaux d'horloges ${ }^{1} \mathrm{~S}_{0}$ et ${ }^{3} \mathrm{P}_{1}$, et donc annulé de la fréquence de résonance des atomes. Nous avons réalisé la mesure la plus précise de la longueur d'onde magique du ${ }^{87} \mathrm{Sr}$, à $c / \lambda_{l}=368554725(5) \mathrm{MHz}$.

Comme les deux états de la transition d'horloge ont un moment cinétique orbital nul $(J=0)$, cette longueur d'onde magique ne dépend pas a priori de la polarisation du réseau optique. Toutefois, l'isotropie de ces états est légèrement brisée par le spin nucléaire $I=9 / 2$ non nul de l'isotope ${ }^{87} \mathrm{Sr}$. Ce spin nucléaire non nul, en plus de faiblement autoriser la transition d'horloge, introduit donc une légère dépendance du déplacement lumineux en fonction de la polarisation du réseau optique : ce sont le déplacement lumineux vectoriel - proportionnel à $m_{F}$ et à l'ellipticité de la polarisation - et le déplacement lumineux tensoriel - quadratique en $m_{F}$ et extrémal pour une polarisation linéaire alignée ou orthogonale au champ magnétique de biais. Du fait de leur origine, le rapport entre ces deux effets et le déplacement lumineux scalaire décrit ci-dessus est de l'ordre de grandeur du rapport entre l'écart hyperfin et les transitions optiques, soit $10^{-4}$. Nous avons mesuré pour la première fois ces effets pour les horloges à réseau optique au strontium, et démontré qu'ils peuvent être contrôlés mieux que $10^{-17}$ à une profondeur de piégeage de $100 E_{R}$ [18]. Nous avons par la suite calculé une estimation théorique de ces effets qui corrobore la mesure expérimentale, et identifié que le déplacement vectoriel (resp. tensoriel) provient principalement du mélange entre l'état excité d'horloge ${ }^{3} \mathrm{P}_{0}$ et l'état ${ }^{3} \mathrm{P}_{1}$ (resp. $\left.{ }^{3} \mathrm{P}_{2}\right)$ [19].

Les déplacements lumineux scalaire, vectoriel et tensoriels, proportionnels à l'intensité du réseau optique, résultent du premier ordre de l'interaction dipolaire électrique. D'autres effets sont à prendre en compte pour complètement évaluer l'effet du piégeage. D'abord, les effets d'ordre élevés (interaction dipolaire magnétique et quadrupolaire électrique) sont estimés à $10^{-17}$ pour $100 E_{R}$. Nous avons pu conforter cette estimation en mesurant une borne supérieure pour ces deux effets [18]. Enfin, des transitions à deux photons partant de l'état excité d'horloge vers des états électroniques de plus haute énergie sont responsables d'un déplacement lumineux qui varie quadratiquement avec l'intensité de piégeage. C'est l'hyper-polarisabilité, que nous avons pu résoudre expérimentalement [20] (fig. 6).

En pratique, les effets de déplacement lumineux scalaires et d'hyper-polarisabilité dépendent de la polarisation du laser de piégeage. Ils sont donc susceptible de fluctuer avec le temps. C'est pourquoi nous mesurons régulièrement la dépendance de la fréquence d'horloge en fonction de la profondeur de piégeage, comme montré figure 6. L'ajustement de cette dépendance avec une parabole donne la correction à appliquer à la fréquence de l'horloge. L'incertitude statistique sur cette estimation est typiquement de $2,5 \times 10^{-17}$.

Enfin, il est nécessaire de prendre en compte l'ensemble de la lumière qui est émise par la source utilisée pour générer le réseau optique, notamment en dehors du spectre d'émission laser. Ce fond spectral peut se situer à plusieurs centaines de nm de la longueur d'onde magique, où même une faible puissance peut induire un large effet systématique. Quantitativement, il est nécessaire que toute la puissance située hors de l'émission laser soit $80 \mathrm{~dB}$ plus faible que cette dernière pour atteindre une exactitude de $10^{-18}$. Cette condition est satisfaite pour notre source - un laser titane saphir - mais 


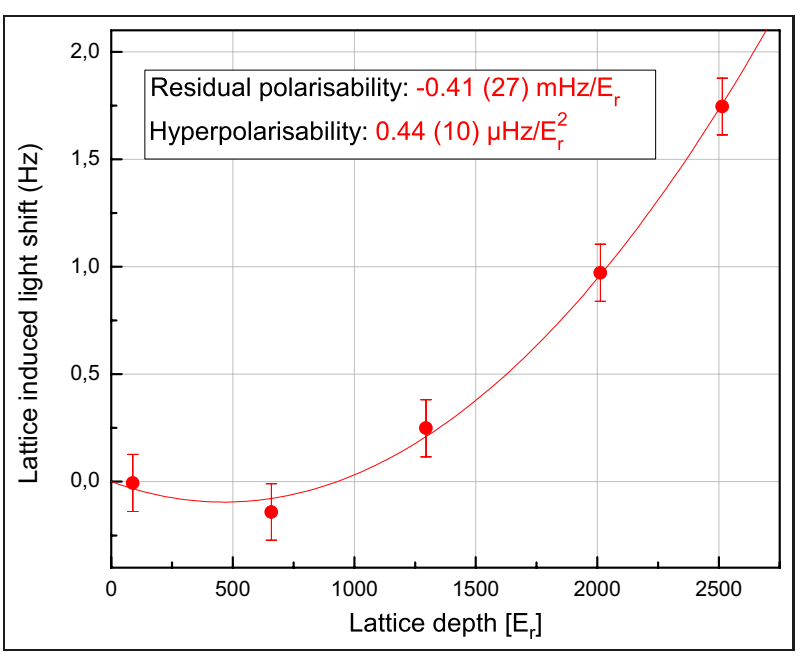

Fig. 6. - Dépendance de la fréquence de résonance de l'horloge $\mathrm{Sr} 2$ en fonction de la profondeur du réseau optique. On observe un comportement parabolique provenant de l'hyperpolarisabilité des niveaux d'horloge. La pente à l'origine indique un désaccord entre la longueur d'onde du réseau optique et la longueur d'onde magique effective qui annule les effets de polarisabilité scalaires et tensoriels, linéaire avec la profondeur de piégeage.

nous avons pu montrer que les sources semi-conducteurs peuvent induire un déplacement de fréquence de plus de $10^{-15}[20]$.

\subsection{Collisions entre atomes froids}

Le déplacement de fréquence induit par les collisions entre atomes froids est un autre effet caractéristique des horloges à réseau optique dans lesquelles un grand nombre d'atomes sont confinés dans un piège. Cet effet a notamment été observé dans les horloges à réseau optique utilisant $\mathrm{du}{ }^{87} \mathrm{Sr}$ avec un réseau unidimensionnel, au niveau de quelques $10^{-16}$ [21]. Avec la méthode du drain atomique décrite ci-dessus, cet effet est néanmoins fortement réduit sur les horloges du LNE-SYRTE. En effet, les atomes sont répartis dans les sites du réseau avec un profil d'écart type de $0,7 \mathrm{~mm}$ qui correspond à la taille du PMO. Ainsi, les sites du réseau optique les plus peuplés comprennent en moyenne 2 à 3 atomes. Les autres groupes utilisent une méthode de chargement fondée sur un deuxième $\mathrm{PMO}$ accordé sur la transition ${ }^{1} \mathrm{~S}_{0} \rightarrow{ }^{3} \mathrm{P}_{1}$ de taille typiquement 10 fois plus faible, avec un réseau optique dont la section transverse est également plus petite. Ainsi, on s'attend à ce que l'effet des collisions soit très inférieur à $10^{-17}$ sur nos horloges. Pour confirmer cette estimation, nous avons mesuré une borne supérieure pour le déplacement de fréquence dû aux collisions en alternant la configuration nominale d'horloge, et une situation dans laquelle le nombre d'atomes par site est deux fois plus élevé. La différence de fréquence entre ces deux configurations est compatible avec zéro, avec une incertitude statistique de $8 \times 10^{-18}$, excluant ainsi un déplacement de fréquence dû aux collisions à ce niveau.

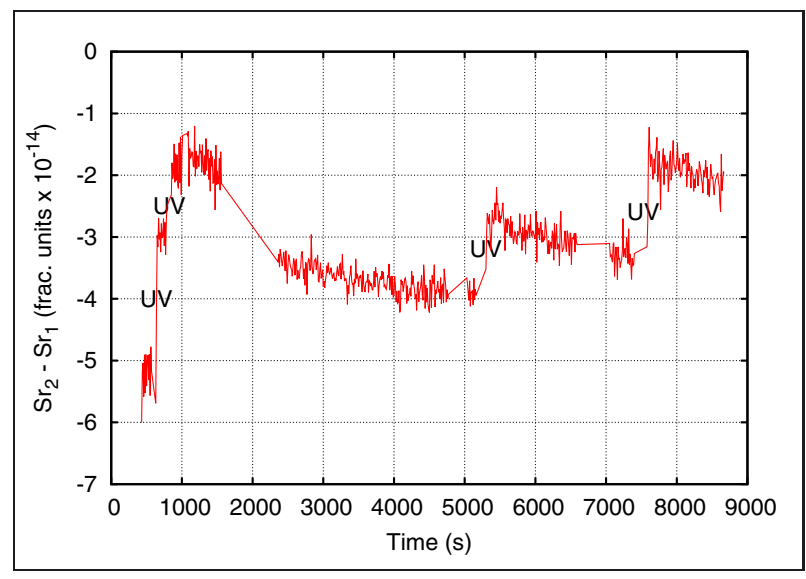

Fig. 7. - Écart de fréquence entre les horloges $\mathrm{Sr} 2$ et $\mathrm{Sr} 1$ en présence de charges statiques sur la première. L'illumination de l'enceinte à vide provoque une réduction de l'écart par détachement des charges. Après plusieurs heures d'illumination avec une LED UV centrée à $365 \mathrm{~nm}$, l'effet des charges statiques est annulé au niveau de $10^{-18}$.

\subsection{Charges électriques statiques}

L'énergie des niveaux électroniques impliqués dans la transition d'horloge dépend du champ électrique statique au niveau des atomes via l'effet Stark statique. Cet effet résulte dans un déplacement de fréquence négatif qui dépend quadratiquement de l'amplitude du champ électrique. Cet effet est important, puisqu'un champ électrique de $1 \mathrm{kV} / \mathrm{m}$ induit un décalage de fréquence relatif de $6 \times 10^{-15}$. Cet effet est supprimé si les atomes sont entourés de surfaces conductrices annulant le champ électrique. Cet effet est connu dans les horloges à ions pour lesquelles des surfaces isolantes sont placées à proximité des atomes pour isoler les électrodes de piégeage [22]. Dans les horloges à réseau optique, de nombreux accès optiques non conducteurs sont nécessaires pour manipuler les atomes, et peuvent ainsi occasionner un déplacement Stark. Nous avons mis en évidence pour la première fois cet effet dans une horloge à réseau optique, en exhibant un écart de fréquence de $10^{-14}$ entre Sr1 et Sr2 [23]. Un modèle capacitif de l'enceinte à vide entourant les atomes nous a permis de localiser les charges sur les miroirs de la cavité optique formant le réseau optique. Nous avons pu annuler le déplacement de fréquence en déchargeant les miroirs avec de la lumière UV (fig. 7). Enfin, nous avons caractérisé le résidu de charge électrique en induisant un champ électrique alternatif à l'aide d'une électrode. Par cette méthode, nous avons montré qu'après décharge l'effet est inférieur à $1,5 \times 10^{-18}$.

\subsection{Rayonnement du corps noir}

Le rayonnement du corps noir émis par l'environnement des atomes déplace les niveaux d'énergie des atomes. Parce qu'à température ambiante ce rayonnement est dans le domaine infrarouge, c'est-à-dire à des fréquences beaucoup plus faibles que les principales résonances électroniques loin des atomes, ce champ est en grande partie assimilable à un champ électrique statique 


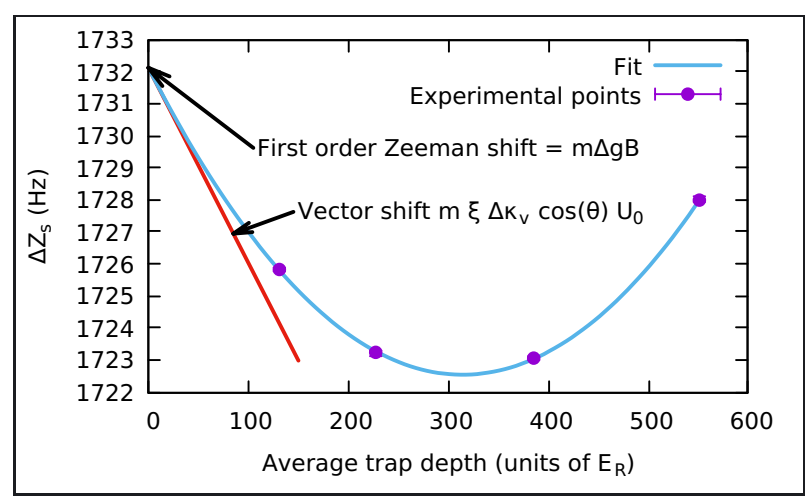

Fig. 8. - Écart de fréquence $Z_{s}$ entre les résonances ${ }^{1} \mathrm{~S}_{0} m_{F}=$ $9 / 2 \rightarrow{ }^{3} \mathrm{P}_{0} m_{F}=9 / 2$ et ${ }^{1} \mathrm{~S}_{0} m_{F}=-9 / 2 \rightarrow{ }^{3} \mathrm{P}_{0} m_{F}=-9 / 2$ en fonction de la profondeur de piégeage. Les barres d'erreurs sur les points sont petites à l'échelle de la figure. L'extrapolation à profondeur nulle donne une évaluation du champ magnétique statique au niveau des atomes. La dépendance quadratique provient du terme de polarisabilité vectoriel décrit dans la paragraphe 4.1. L'ajustement de cette dépendance fournit une information sur la géométrie du réseau [19].

d'amplitude $872 \mathrm{~V} / \mathrm{m}$ à une température de $300 \mathrm{~K}$. En utilisant une mesure précise de la polarisabilité différentielle pour la transition d'horloge effectuée à la PTB [24], on en déduit le déplacement de fréquence Stark associé. Une correction supplémentaire de $7 \%$ doit être appliquée pour prendre en compte la nature ondulatoire du champ rayonné [6], portant la correction à $5,2 \times 10^{-15}$ pour l'horloge Sr2. L'incertitude finale sur l'estimation du déplacement de fréquence dû au rayonnement du corps noir provient alors de l'incertitude sur la température de l'enceinte à vide placée autour des atomes, combinée à l'incertitude sur le terme de polarisabilité dynamique. Six thermomètres à résistance de platine (PT100), étalonnés par le Laboratoire national de métrologie et d'essais (LNE), relèvent la température au point le plus chaud et au point le plus froid de l'enceinte à vide. Un écart maximal de $0,8 \mathrm{~K}$ est typiquement mesuré, correspondant à une incertitude type de $0,23 \mathrm{~K}$. À partir de cette valeur, l'incertitude totale sur le déplacement de fréquence dû au rayonnement du corps noir est alors de $1,8 \times 10^{-17}$.

\subsection{Effets Zeeman linéaire et quadratique}

L'interaction entre les atomes et le champ magnétique induit un effet Zeeman linéaire avec le champ magnétique et le nombre quantique $m_{F}$, ainsi qu'un déplacement de fréquence quadratique avec le champ magnétique. L'effet linéaire est éliminé en définissant la fréquence de l'horloge comme la moyenne entre les fréquences des résonances ${ }^{1} \mathrm{~S}_{0} m_{F}=9 / 2 \rightarrow{ }^{3} \mathrm{P}_{0} m_{F}=9 / 2$ et ${ }^{1} \mathrm{~S}_{0} m_{F}=$ $-9 / 2 \rightarrow{ }^{3} \mathrm{P}_{0} m_{F}=-9 / 2$. Pour mesurer cette moyenne, le système d'asservissement de la fréquence du laser d'horloge sonde alternativement les deux résonances; la différence $Z_{s}$ entre les fréquences des deux transitions donne quant à elle une mesure du champ magnétique à la position des atomes (fig. 8), en connaissant les facteurs de Landé des niveaux d'horloges [19].
L'effet quadratique doit être estimé puis compensé. C'est, avec l'effet du rayonnement du corps noir, l'effet systématique le plus grand pour les horloges à réseau optique au strontium. Pour un champ magnétique de $155 \mu \mathrm{T}$ usuellement appliqué sur nos horloges, le déplacement de fréquence est de $1,317 \times 10^{-15}$. Pour estimer la correction de fréquence $-\delta v$ à appliquer, nous avons mesuré une courbure de l'effet Zeeman quadratique de $\delta v / Z_{s}^{2}=-0,246(2) \mathrm{Hz} / \mathrm{kHz}^{2}$ [18]. L'incertitude sur cette correction, de $1,2 \times 10^{-17}$, provient principalement de l'incertitude sur cette courbure.

\subsection{Dérive de phase dans les modulateurs acousto-optiques}

Pendant le cycle d'horloge, le laser d'horloge est commuté par un modulateur acousto-optique (MAO). Lors de la mise en marche du modulateur, un transitoire thermique provoque une variation temporelle de l'indice du cristal du modulateur, et donc un déplacement de fréquence qui introduit un biais de fréquence. Nous avons mesuré cet effet de deux manières. D'abord, par une mesure interférométrique hétérodyne, puis en mesurant la fréquence de l'horloge. Dans ces deux cas, le biais de fréquence dépend linéairement de la puissance, et dépend de l'alignement du faisceau laser dans le modulateur. En augmentant la puissance du faisceau laser et en diminuant la puissance électrique envoyée dans le modulateur, on peut aisément réduire l'effet à quelques $10^{-18}$.

\section{7. $\quad$ Autres effets}

Plusieurs autres effets systématiques, de moindre importance, ont été évalués :

La distorsion de la résonance d'horloge due à la présence d'autres résonances voisines (par exemple venant de l'excitation d'états magnétiques $m_{F} \neq \pm 9 / 2$ résiduels) a été évaluée avec une incertitude statistique de $2 \times 10^{-17}$ en faisant varier la largeur de la résonance.

Le flux de puissance du rayonnement du corps noir issu de la source de strontium chauffée à $860 \mathrm{~K}$ est délicat à estimer avec précision du fait des réflexions de ce rayonnement sur les parois de l'enceinte à vide. Un modèle pessimiste de l'effet permet de majorer son incertitude par $8 \times 10^{-18}$.

La dérive systématique du laser d'horloge, typiquement inférieure à $2 \mathrm{mHz} / \mathrm{s}$, prise entre deux cycles d'horloge successifs introduit un biais dans le calcul du signal d'erreur. Compte tenu du temps de cycle typique $T_{c}=700 \mathrm{~ms}$, cette dérive correspond à un effet systématique borné par $3 \times 10^{-18}$.

L'effet systématique résultant des collisions avec les atomes chauds peut être borné en dessous de $5 \times 10^{-18}$ à partir de la donnée du temps de vie des atomes dans le piège (de l'ordre de $0,3 \mathrm{~s}$ ) [25].

Le déplacement lumineux associé au laser d'horloge peut être évalué à partir d'un calcul théorique de la 
polarisabilité dynamique des niveaux d'horloge. On estime ainsi un effet inférieur à $4 \times 10^{-19}$.

\section{Comparaisons d'horloges}

Les comparaisons d'horloges sont un élément clé dans l'exploitation des horloges atomiques. D'abord, elles sont nécessaires pour confirmer les performances affichées : la comparaison entre deux horloges donne directement la stabilité de fréquence combinée de ces deux horloges. De plus, si les deux horloges utilisent la même espèce atomique, l'égalité entre leurs fréquences confirmera le bilan d'exactitude. Si les horloges utilisent deux espèces atomiques différentes, la reproductibilité dans le temps et l'espace du rapport de fréquence entre les horloges devra être compatible avec le bilan d'exactitude combiné des horloges.

Ensuite, ce sont des comparaisons entre horloges que découlent la plupart de leurs applications. Deux d'entre elles sont déjà en cours d'exploration dans les instituts nationaux de métrologie, en particulier au LNE-SYRTE. Il s'agit de détecter une hypothétique dérive des constantes fondamentales, et d'explorer les variations spatiales du potentiel gravitationnel de la Terre.

Les horloges optiques posent un nouveau défi pour les comparaisons d'horloges distantes. En effet, les méthodes satellitaires couramment employées pour comparer les horloges micro-ondes, principalement fondées sur les signaux des systèmes de géo-localisation (comme le GPS), n'ont pas une résolution statistique permettant de tirer parti de la stabilité et de l'exactitude des horloges optiques. À ce jour, aucune méthode satellitaire ne permet de comparer des horloges distantes à mieux que l'exactitude des étalons primaires au césium (c'est-à-dire quelques $10^{-16}$ ).

\subsection{Comparaisons locales de deux horloges au strontium}

Le LNE-SYRTE a été le premier laboratoire à développer et comparer deux horloges à réseau optique. En 2013, nous avons mesuré un écart de fréquence entre ces deux horloges au strontium compatible avec le bilan d'incertitude combiné des deux horloges, au niveau de $1,4 \times 10^{-16}$ [26]. Cet accord a permis de confirmer le bilan d'incertitude des horloges, et donne une indication forte que les effets systématiques des horloges à réseau optique sont effectivement maîtrisés avec une meilleure incertitude que les horloges micro-onde.

Cette comparaison a montré que les comparaisons locales entre horloges de même nature sont essentielles : au cours de la caractérisation des horloges, la mesure de la différence de fréquence entre les horloges nous a permis d'identifier deux effets systématiques qui n'avaient pas été pris en compte par le passé pour les horloges à réseau optique, et qui peuvent induire des déplacements de fréquence supérieurs à $10^{-15}$. Ces deux effets sont le déplacement de fréquence dû à la présence de charges statiques (paragraphe 4.3) et le déplacement lumineux in-

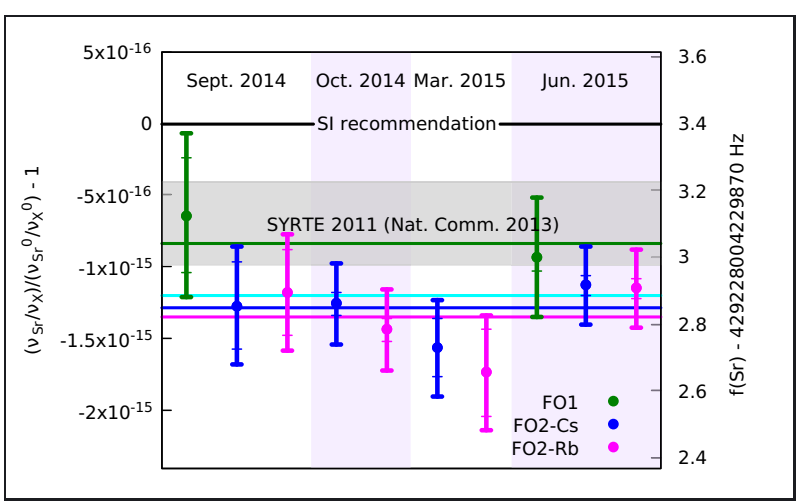

Fig. 9. - Rapport de fréquence entre la résonance d'horloge du strontium et les fréquences des horloges au césium et au rubidium, relativement aux rapports recommandés par le comité consultatif temps fréquence (CCTF) en 2012 (labellisés ${ }^{0}$ ). Les horloges impliquées dans cette mesure sont la fontaine atomique au césium FO1 et la fontaine mixte au rubidium et au césium FO2 [2,27]. Pour les comparaisons entre les horloges au strontium et au césium, l'échelle de droite indique la fréquence absolue correspondante pour la fréquence de l'horloge au strontium dans le SI. Les barres d'erreur grasses indiquent l'incertitude totale. La fiabilisation des horloges au strontium a permis de mesurer ces rapports de fréquences sur de longues périodes (voir paragraphe 6.1), réduisant ainsi considérablement la barre d'erreur statistique (barre fine). À titre de comparaison, le graphique indique la recommandation du CCTF pour la fréquence de l'horloge au strontium, ainsi qu'une précédente mesure datant de 2011 et publiée en 2013 par notre groupe [26].

duit par le spectre incohérent issu des sources laser semiconducteurs (paragraphe 4.1).

\subsection{Comparaisons locales d'horloges d'espèces différentes}

Le LNE-SYRTE dispose d'un ensemble d'horloges atomiques micro-onde [27] et une horloge optique au mercure [2], qui peuvent être comparées aux horloges au strontium via un peigne de fréquences généré par un laser femto-seconde fibré [28]. Cet ensemble d'horloges nous a permis de réaliser des mesures répétées du rapport de fréquence entre l'horloge au strontium et les étalons primaires au césium. La stabilité de ces comparaisons, de l'ordre de $3 \times 10^{-14} / \sqrt{\tau}$ est limitée par le bruit de projection quantique des horloges de type fontaines atomiques (FO) ; l'exactitude, de $2,6 \times 10^{-16}$, est également limitée par l'exactitude des fontaines (fig. 9). Ces mesures, qui constituent la meilleure détermination d'une fréquence absolue à ce jour, sont reproductibles sur plusieurs années avec un écart inférieur à l'incertitude systématique, ce qui indique une excellente maitrise des effets systématiques sur les deux horloges. De plus, elles sont en très bon accord avec des mesures similaires effectuées à la PTB [29,30] (voir également paragraphe 5.6). Plus récemment, nous avons mesuré pour la première fois le rapport de fréquence entre les horloges au strontium et au rubidium, avec une incertitude comparable (fig. 9). Enfin, les premières comparaisons tout optique entre les horloges à réseau optique au strontium et au mercure 


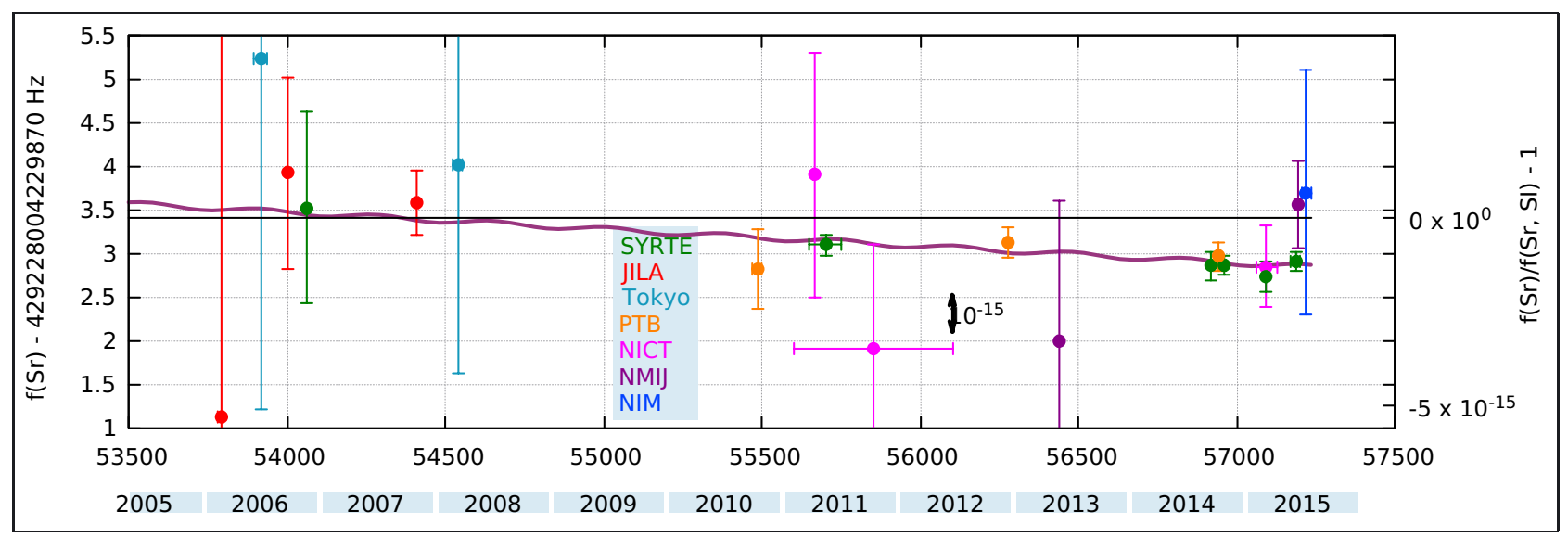

Fig. 10. - Mesures du rapport de fréquence optique - micro-onde entre les horloges au strontium et au césium, par le LNE-SYRTE [26,35], le JILA (Colorado, États-unis) [36,37], la PTB (Allemagne) [29, 30,38], l’Université de Tokyo (Japon) en 2006 et 2008 [39], le NICT (Japon) [40-42], le NMIJ (Japon) [43,44] et le NIM en 2015 (Chine) [45]. Un ajustement de ces mesures par une dérive linéaire dans le temps superposée à une variation sinusoïdale d'amplitude $a$ calée sur la rotation de la Terre autour du soleil permet de contraindre la dérive du rapport de fréquence par $d \ln \left(v_{\mathrm{Sr}} / v_{\mathrm{Cs}}\right) / d t=-(1,7 \pm 0,7) \times 10^{-16} /$ an et $a=(0,7 \pm 1,8) \times 10^{-17}$. De futures mesures permettront de réduire encore davantage l'incertitude sur ces dérives.

conduites au LNE-SYRTE permettent de s'affranchir des étalons micro-onde, et ainsi de démontrer une stabilité de fréquence dix fois meilleure, à quelques $10^{-15} / \sqrt{\tau}$.

\subsection{Comparaisons intracontinentales}

Les comparaisons d'horloges distantes, par exemple en vue de réaliser des échelles de temps internationales, utilisent à ce jour des méthodes satellitaires. Pourtant, même les meilleures d'entre elles ne permettent pas de comparer des horloges avec une résolution meilleure que quelques $10^{-16}$ après plusieurs semaines [31], et donc $a$ fortiori de tirer parti de la stabilité offerte par les horloges à réseau optique. L'horloge $\mathrm{Sr} 2$ a été impliquée dans deux campagnes de comparaisons d'horloges par satellite, dans le cadre du projet européen ITOC [32]. L'une a duré 7 jours en octobre 2014 et l'autre 21 jours en juin 2015. Le rapport de fréquence entre Sr2 et l'horloge optique à ion $\mathrm{Yb}^{+}$de la PTB a pu être mesuré avec une incertitude de l'ordre de $5 \times 10^{-16}$, avec un résultat en accord avec les mesures indépendantes via les fontaines atomiques au césium.

Pour comparer des horloges à réseau optique distantes sans dégrader leur stabilité de fréquence, de nouveaux moyens de comparaison utilisant des fibres optiques dont les fluctuations d'indice sont compensées ont été mises en place et caractérisées [33]. Un lien connectant le LNE-SYRTE et la PTB (Braunschweig, Allemagne) long de $1415 \mathrm{~km}$ et récemment mis en place a permis de comparer les horloges à réseau optique au strontium des deux instituts. La stabilité de la comparaison est entièrement limitée par les horloges, et a permis de démontrer une résolution statistique de $2 \times 10^{-17}$ après $1 \mathrm{~h}$ d'intégration [34]. Cette résolution est un ordre de grandeur meilleure que celle offerte par les méthodes satellitaires, atteinte après un temps d'intégration deux ordres de grandeur plus petit. De plus, les performances du lien sont compatibles avec les progrès attendus pour les horloges à réseau optique dans la gamme des $10^{-19}$. La différence de fréquence entre les deux horloges est $(4,7 \pm 5,0) \times 10^{-17}$, compatible avec zéro. C'est la première comparaison dans la gamme des $10^{-17}$ entre deux horloges à réseau optique complètement indépendantes, développées par des équipes différentes. Cette expérience démontre donc pleinement la reproductibilité des horloges à réseau optique.

\subsection{Comparaisons intercontinentales}

Le strontium est l'espèce atomique la plus couramment choisie pour les horloges à réseau optique, notamment du fait de l'accessibilité des sources laser nécessaires au refroidissement et au piégeage des atomes. Ainsi, les horloges au strontium du LNE-SYRTE ont pu être indirectement comparées aux autres horloges au strontium développées dans le monde via les étalons primaires de fréquence au césium. Sept laboratoires ont publié des mesures du rapport de fréquence entre les horloges au strontium et au césium, toutes en très bon accord (fig. 10).

À ce jour, il n'existe pas de moyen de comparaison permettant de comparer des horloges optiques à l'échelle mondiale avec une résolution meilleure que $10^{-16}$. La mission spatiale Pharao/ACES [46], au cours de laquelle une horloge avec des atomes froids de césium sera embarquée sur la station spatiale internationale, offrira cette opportunité à partir du printemps 2017, date prévue pour son lancement. Les horloges au strontium du LNESYRTE feront partie du segment sol de cette mission.

\subsection{Mesure du potentiel gravitationnel et applications en géodésie}

La théorie de la relativité générale dit que la fréquence d'une horloge dépend du potentiel gravitationnel 
dans lequel elle est située. C'est l'effet de « déplacement gravitationnel vers le rouge ». Ainsi, une horloge dont l'altitude augmente de $1 \mathrm{~m}$ voit sa fréquence augmenter relativement de $10^{-16}$ [47]. Comparer des horloges distantes permet donc de mesurer la différence de potentiel gravitationnel entre ces horloges, qui provient majoritairement du potentiel gravitationnel terrestre [48]. Les comparaisons internationales d'horloges pourront donc apporter des informations sur la structure interne de la Terre. Elles viennent en complément des mesures du champ gravitationnel, davantage sensibles aux variations de gravité aux courtes échelles du fait de la dépendance en $1 / r^{2}$ du champ gravitationnel, et des méthodes d'orbitographie de satellites capables de mesurer le potentiel gravitationnel à plus grande échelle.

À titre d'exemple, la comparaison entre les horloges à réseau optique au strontium du LNE-SYRTE et de la PTB nécessite une correction du déplacement gravitationnel de $(247,4 \pm 0,4) \times 10^{-17}$, obtenue grâce à une modélisation du géoïde, couplée à des relevés topographiques sur le terrain. L'incertitude sur cette correction, qui correspond à une incertitude de $4 \mathrm{~cm}$ sur l'altitude gravitationnelle des horloges, est encore inférieure à l'exactitude de nos horloges. Toutefois, il apparaît prévisible que la prochaine génération d'horloges à réseau optique aura une exactitude meilleure que $10^{-18}$. Ces horloges pourront donc être utilisées pour raffiner les modèles géodésiques.

\subsection{Applications en physique fondamentale}

La fréquence de résonance ne dépend que des lois fondamentales de la physique et de leurs paramètres, tels la constante de structure fine $\alpha$ ou les rapports de masse entre les particules fondamentales ${ }^{1}$. Toutefois, si la dépendance explicite de la fréquence de résonance d'un atome en fonction de ces paramètres n'est pas calculable en pratique, la loi de puissance de cette dépendance peut être estimée théoriquement, et diffère pour chaque atome [49]. Ainsi, en bornant l'évolution relative dans le temps du rapport de fréquence entre différentes espèces atomiques, il est possible de borner la variation temporelle relative des constantes fondamentales. De telles variations seraient la signature de théories physiques allant au-delà du modèle standard des particules (dérive linéaire des constantes) ou de la relativité générale (variation des constantes en fonction du potentiel gravitationnel du Soleil, qui briserait le principe d'équivalence). La recherche en laboratoire d'une variation des rapports de fréquence entre espèces atomiques [50], qui traduirait une dérive contemporaine des constantes fondamentales, est en particulier motivée par des tests astronomiques qui pourraient avoir détecté de telles variations sur une échelle de temps de l'ordre de l'âge de l'univers [51]

Du fait qu'elle a été reproduite un grand nombre de fois sur plusieurs années, la mesure du rapport

\footnotetext{
1 Cette situation est différente de celle des autres oscillateurs (pendule, quartz, la Terre...), dont la fréquence est contingente.
}

de fréquence entre les horloges au strontium et au césium montrée figure 10, permet de significativement contribuer à borner la dérive des constantes fondamentales $[26,37,50]$.

\section{Vers une redéfinition de la seconde fondée sur une transition optique}

L'atome de césium est actuellement utilisé comme base dans la définition de la seconde du Système international d'unités (SI). Comme les horloges au césium sont maintenant surpassées par les horloges optiques, il est envisagé de redéfinir l'unité de temps sur la base d'une horloge optique. De nombreuses transitions optiques, dont plusieurs sont déjà recommandées par le Comité international des poids et mesures (CIPM) comme représentations secondaires de la seconde, pourront être choisies pour cette redéfinition. Parmi ces transitions optiques, celle du strontium est un bon candidat car sa réalisation pratique est parmi les plus simples, et il est à ce jour l'étalon de fréquence optique le plus répandu et le mieux reproduit dans les laboratoires de métrologie.

\subsection{Horloges au strontium opérationnelles}

La contribution aux échelles de temps internationales, notamment au TAI est une première étape vers la redéfinition de la seconde avec un étalon optique. Une telle contribution consiste en la calibration d'un oscillateur local - en l'occurrence un maser à hydrogène - réalisant une échelle de temps locale - pour le LNE-SYRTE, il s'agit d'UTC(OP). Pour qu'une telle contribution soit représentative, il faut d'une part des mesures longues capables de calibrer l'oscillateur local en continu sur plusieurs jours, et d'autre part des mesures répétées pour avoir un poids significatif. Ainsi, il est nécessaire de faire fonctionner les horloges optiques sur des intervalles de 5 à 10 jours, correspondant à des plages de calibration du TAI fixées par le Bureau International des Poids et Mesures, avec un taux de remplissage typique supérieur à $80 \%$ pour pouvoir détecter de façon fiable les fluctuations de fréquence de l'oscillateur. C'est pourquoi une telle contribution nécessite la démonstration d'horloges opérationnelles.

Nous avons effectué un travail de fiabilisation des horloges au strontium du LNE-SYRTE afin de pouvoir réaliser cet objectif. Ce développement s'est conclu par la démonstration de mesures longues et quasi-continues, avec une assistance humaine limitée. La figure 11 montre une telle mesure, d'une durée de 10 jours avec un taux de remplissage de $93,8 \%$. Cette mesure est donc compatible avec une contribution au TAI. Nous avons répété cette démonstration en juin 2015, sur trois semaines avec un taux de remplissage de $83 \%$ [52]. L'ensemble de ces mesures seront prochainement répétées, et pourront constituer une première contribution d'une horloge optique au TAI. 


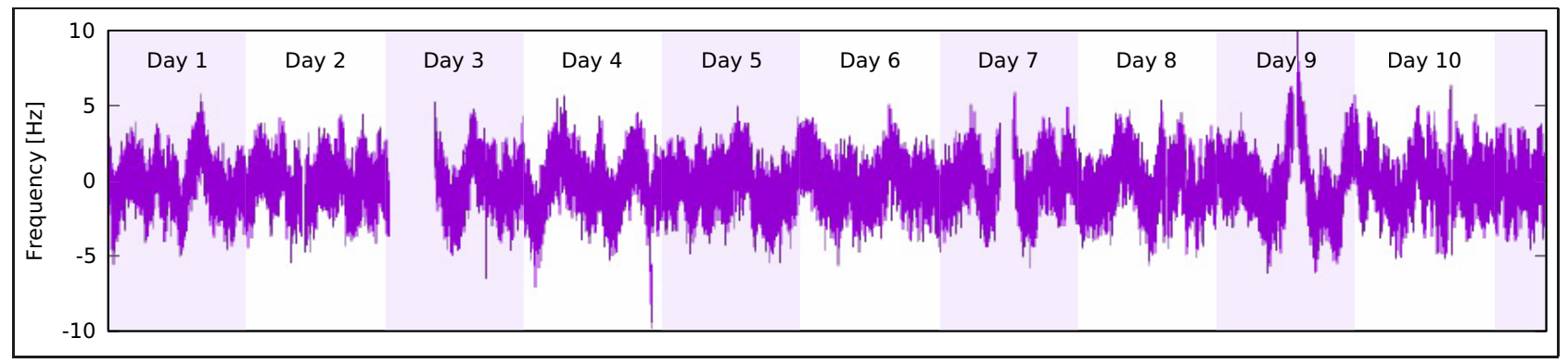

Fig. 11. - En octobre 2014, l'horloge Sr2 a fonctionné pendant 10 jours avec un taux de remplissage de 93,8 \%. Cette figure montre la différence de fréquence entre l'horloge au strontium et la cavité ultrastable de référence de l'horloge (la dérive polynomiale de la cavité est retranchée de ces données, si bien que les fluctuations résiduelles montrent le bruit thermique de la cavité). Parmi ces données, on trouve un intervalle de 90 heures consécutives avec un remplissage de 99,7 \%, comprenant un intervalle nocturne de 7 heures avec un remplissage de $100 \%$.

\section{Conclusion}

Les horloges à réseau optique, apparues au début des années 2000, ont maintenant largement surpassé les horloges micro-onde, et mènent, encore talonnées par les horloges optiques à ion, la course à l'exactitude. Le développement de nombreuses horloges à réseau optique à travers le monde a démontré leur reproductibilité dans la gamme des $10^{-17}$, donnant une solide crédibilité aux performances annoncées. À court terme, les horloges à réseau optique devraient contribuer à l'élaboration d'échelles de temps internationales, ce qui est une première étape vers une redéfinition de la seconde, unité de temps dans le SI.

Pourtant, malgré ces rapides avancées, les performances ultimes de ces horloges sont encore floues. Les progrès attendus dans la stabilisation de la fréquence des lasers, avec des cavités de référence cryogéniques [53,54] ou des méthodes de stabilisation innovantes [55], laissent entrevoir des stabilités au niveau du bruit de projection quantique, de l'ordre de $10^{-17} / \sqrt{\tau}$. Avec une telle stabilité, l'évaluation des effets systématiques au niveau de $10^{-19}$ est tout à fait envisageable. À un tel niveau de performances, les applications des horloges à réseau optique en géodésie et en physique fondamentale seront possibles.

\section{Remerciements}

Les travaux décrits dans cet article ont été réalisés au LNE-SYRTE, Observatoire de Paris, PSL Research University, CNRS, Sorbonne Universités, UPMC Univ. Paris 06. Ils ont reçu un soutien financier de ses tutelles, ainsi que du Centre National d'Études Spatiales (CNES), du Conseil Régional Île-de-France (DIM IFRAF et Nano'K), du European Metrology Research Programme (Contrats T1 J2.1 OCS, EXL-01 QESOCAS et SIB-55 ITOC), de l'Agence Nationale de la Recherche (Labex First-TF ANR-10-LABX-48-01), et le l'European Space Agency (projet SOC) et du projet FP7 SOC2. Les travaux ont bénéficié de nombreuses contributions des services informatique, électronique, mécanique et administratif du SYRTE. Le laboratoire SYRTE, SYstèmes de Référence Temps-Espace, est UMR 8630. Il est membre du Labex FIRST-TF, des DIM IFRAF et Nano'K. Le LNE, Laboratoire national de métrologie et d'essais, est l'institut national de métrologie français.

\section{Références}

[1] Guéna J., Abgrall M., Rovera D., Laurent P., Chupin B., Lours M., Santarelli G., Rosenbusch P., Tobar M., Li R., Gibble K., Clairon A. et Bize S. "Progress in atomic fountains at LNE-SYRTE". IEEE Trans. Ultrasonics, Ferroelectrics, and Frequency Control, 59(3), 2012, 391-409.

[2] Abgrall M., Chupin B., Sarlo L.D., Guéna J., Laurent P., CoQ Y.L., TARgat R.L., Lodewyck J., LOURS M., Rosenbusch P., Rovera G.D. et BIZE S. "Atomic fountains and optical clocks at SYRTE: Status and perspectives". Comptes Rendus Physique, 16(5), $2015,461-470$.

[3] Ludlow A.D., Boyd M.M., Ye J., PeIK E. et SchMidT P.O. "Optical atomic clocks". Rev. Mod. Phys., 87, 2015, 637-701.

[4] Hinkley N., Sherman J.A., Phillips N.B., Schioppo M., Lemke N.D., Beloy K., Pizzocaro M., OATeS C.W. et LudLow A.D. "An Atomic Clock with $10^{-18}$ Instability”. Science, 341(6151), 2013, 1215-1218.

[5] Bloom B.J., Nicholson T.L., Williams J.R., CAMPBell S.L., Bishof M., Zhang X., Zhang W., BROMLEY S.L. et YE J. "An optical lattice clock with accuracy and stability at the $10^{-18}$ level". Nature, 506, 2014, 12941.

[6] Nicholson T., CAMpbell S., Hutson R., Marti G., Bloom B., McNally R., Zhang W., Barrett M., SAFronova M., Strouse G. et al.. "Systematic evaluation of an atomic clock at $2 \times 10^{-18}$ total uncertainty". Nat. commun., 6, 2015, 6896.

[7] HäFner S., FAlke S., Grebing C., Vogt S., Legero T., MerimaA M., Lisdat C. et SterR U. " $8 \times 10^{-17}$ fractional laser frequency instability with a long roomtemperature cavity”. Opt. Lett., 40(9), 2015, 2112-2115.

[8] Ushijima I., Takamoto M., Das M., OhKubo T. et Katori H. "Cryogenic optical lattice clocks". Nat. Photon., 9, 2015, 185.

[9] Baillard X., Le Targat R., Fouché M., Brusch A., WESTERGAARD P.G., LECALlier A., LODEWYCK J. et LEMONDE P. "Horloge à réseau optique à atomes de strontium”. Revue Française de Métrologie, 17, 2008, 11-27. 
[10] ITANO W.M., BERGQUIST J.C., BOllingeR J.J., GILligan J.M., HeINZEN D.J., Moore F.L., RAIZEN M.G. et WINELAND D.J. "Quantum projection noise: Population fluctuations in two-level systems". Phys. Rev. A, 47, 1993, 3554-3570.

[11] Chou C.W., Hume D.B., KoelemeiJ J.C.J., Wineland D.J. et Rosenband T. "Frequency Comparison of Two High-Accuracy $\mathrm{Al}^{+}$Optical Clocks". Phys. Rev. Lett., 104, 2010, 070802 .

[12] Santarelli G., Audoin C., MaKdissi A., Laurent P., DICK G. et ClaIRON C. "Frequency stability degradation of an oscillator slaved to a periodically interrogated atomic resonator". IEEE Trans. Ultrasonics, Ferroelectrics, and Frequency Control, 45(4), 1998, 887894

[13] WestergaARD P., LODEWyCK J. et Lemonde P. "Minimizing the Dick effect in an optical lattice clock". IEEE Trans. Ultrasonics, Ferroelectrics, and Frequency Control, 57(3), 2010, 623-628.

[14] Lodewyck J., WestergaARd P.G., LeCAllier A., LORINI L. et LEMONDE P. "Frequency stability of optical lattice clocks". New J. Phys., 12(6), 2010, 065026.

[15] Millo J., Magalhães D., Mandache C., Le Coq Y., ENGLISH E., WESTERGAARD P., LODEWYCK J., BIZE S., LEMONDE P. et SANTARELLI G. "Ultrastable lasers based on vibration insensitive cavities". Phys. Rev. A, 79(5), 2009, 053829.

[16] Numata K., Kemery A. et CAMP J. “Thermal-Noise Limit in the Frequency Stabilization of Lasers with Rigid Cavities". Phys. Rev. Lett., 93, 2004, 250602.

[17] Lodewyck J., WestergaARd P.G. et Lemonde P. "Nondestructive measurement of the transition probability in a Sr optical lattice clock". Phys. Rev. A, 79(6), 2009, 061401.

[18] WestergaARD P.G., LODEWYCK J., LORINI L., Lecallier A., Burt E.A., Zawada M., Millo J. et LEMONDE P. "Lattice-Induced Frequency Shifts in $\mathrm{Sr}$ Optical Lattice Clocks at the $10^{-17}$ Level”. Phys. Rev. Lett., 106(21), 2011, 210801.

[19] Shi C., Robyr J.L., EISMANn U., ZaWADA M., LoRINI L., LE TARGAT R. et LODEWYCK J. "Polarizabilities of the ${ }^{87}$ Sr clock transition". Phys. Rev. A, 92, 2015, 012516.

[20] Le TARgat R., Lorini L., Gurov M., Zawada M., GARTMAN R., NAGÓRNY B., LEMONDE P. et LODEWYCK J. "Comparison of two Strontium optical lattice clocks in agreement at the $10^{-16}$ level". "European Frequency and Time Forum (EFTF)", 2012. 19-22.

[21] Swallows M., Martin M., Bishof M., BenKo C., LiN Y., BLATT S., REY A. et YE J. "Operating a ${ }^{87} \mathrm{Sr}$ optical lattice clock with high precision and at high density". IEEE Trans. Ultrasonics, Ferroelectrics, and Frequency Control, 59(3), 2012, 416-425.

[22] Poitzsch M.E., Bergquist J.C., Itano W.M. et WINELAND D.J. "Cryogenic linear ion trap for accurate spectroscopy”. Rev. Sci. Instrum., 67(1), 1996, 129-134.

[23] LODEWYcK J., ZaWADA M., LORINI L., GUROV M. et LEMONDE P. "Observation and cancellation of a perturbing dc stark shift in strontium optical lattice clocks".
IEEE Trans. Ultrasonics, Ferroelectrics, and Frequency Control, 59(3), 2012, 411-415.

[24] Middelmann T., Falke S., Lisdat C. et SterR U. "High accuracy correction of blackbody radiation shift in an optical lattice clock". Phys. Rev. Lett., 109(26), 2012, 263004.

[25] GIBBLE K. "Scattering of Cold-Atom Coherences by Hot Atoms: Frequency Shifts from Background-Gas Collisions". Phys. Rev. Lett., 110, 2013, 180802.

[26] Le Targat R., Lorini L., Le Coq Y., Zawada M., Guéna J., Abgrall M., Gurov M., Rosenbusch P., ROVERA D.G., NAGÓRNY B., GARTMAN R., WESTERGAARD P.G., TOBAR M.E., LOURS M., Santarelli G., Clairon A., Bize S., Laurent P., LEMONDE P. et LODEWYCK J. "Experimental realization of an optical second with strontium lattice clocks". Nat. Commun., 4, 2013, 2109.

[27] Guéna J., Abgrall M., Rovera D., Laurent P., Chupin B., Lours M., SANTARElli G., Rosenbusch P., Tobar M., Li R., Gibble K., Clairon A. et Bize S. "Progress in atomic fountains at LNE-SYRTE". IEEE Trans. Ultrasonics, Ferroelectrics and Frequency Control, 59(3), 2012, 391-410.

[28] Le Coq Y., Le Targat R., Zhang W., Haboucha A., Millo J., Nicolodi D., XU Z., Li T., Boudot R., KeRsalÉ Y. et al.. "Peignes de fréquences femtosecondes pour la mesure des fréquences optiques". Revue française de métrologie, 32, 2012, 35-47.

[29] Falke S., Lemke N., Grebing C., Lipphardt B., Weyers S., GerginOV V., HunTEMANN N., HagemanN C., Al-Masoudi A., HÄFner S., Vogt S., STERR U. et LISDAT C. "A strontium lattice clock with $3 \times 10^{-17}$ inaccuracy and its frequency". New J. Phys., 16(7), 2014, 073023.

[30] Grebing C., Al-Masoudi A., D"ORscher S., HÄFNER S., GERGINOV V., WEYERS S., LIPPHARDT B., RIEHLE F., STERR U. et LISDAT C., "Realization of a timescale with an accurate optical lattice clock"; Optica, 3, 6, 2016, 563569.

[31] Fujieda M., Piester D., Gotoh T., Becker J., AidA M. et BAUCH A. "Carrier-phase two-way satellite frequency transfer over a very long baseline". Metrologia, 51(3), 2014, 253 .

[32] Margolis H., Godun R., GiLl P., Johnson L., SHEMAR S., WhibBerley P., CAlonico D., LeVI F., Lorini L., Pizzocaro M. et al.. "International timescales with optical clocks (ITOC)". "European Frequency and Time Forum \& International Frequency Control Symposium (EFTF/IFC)", IEEE, 2013. 908-911.

[33] Santarelli G., Lopez O., Kéfélian F., Jiang H., Chanteau B., Lours M., Narbonneau F., Haboucha A., Crane S., Daussy C. et al.. "Transfert de fréquence par fibre optique en métrologie Frequency transfer using optical fiber in metrology". Revue française de métrologie, 33, 2014, 9-18.

[34] Lisdat C., Grosche G., Quintin N., Shi C., Raupach S., GREBING C., Nicolodi D., STEFANI F., AL-Masoudi A., DöRSCHER S. et al.. "A clock network for geodesy and fundamental science". arXiv:1511.07735. 
[35] Baillard X., Fouché M., Le TARgat R., WestergaARD P.G., LeCAllier A., Chapelet F., ABGRALl M., Rovera G.D., LAURENT P., ROSENBUSCH P., Bize S., Santarelli G., Clairon A., Lemonde P., Grosche G., LiPPHARDT B. et SchNATZ H. "An optical lattice clock with spin-polarized ${ }^{87} \mathrm{Sr}$ atoms". Eur. Phys. J. D, 48(1), 2008, 11-17.

[36] Campbell G.K., Ludlow A.D., Blatt S., Thomsen J.W., MARTIN M.J., DE MirandA M.H.G., ZELEVINSKY T., BOYD M.M., YE J., DidDAMS S.A., HEAVNER T.P., PARKER T.E. et JEFFERTS S.R. "The absolute frequency of the ${ }^{87} \mathrm{Sr}$ optical clock transition". Metrologia, 45(5), 2008, 539.

[37] Blatt S., Ludlow A.D., Campbell G.K., Thomsen J.W., ZELEVINSKY T., BOYD M.M., YE J., BAILlARD X., Fouché M., Le Targat R., BRUsch A., LEMONDE P., TAKAmoto M., Hong F.L., Katori H. et Flambaum V.V. "New Limits on Coupling of Fundamental Constants to Gravity Using ${ }^{87} \mathrm{Sr}$ Optical Lattice Clocks". Phys. Rev. Lett., 100, 2008, 140801

[38] Falke S., Schnatz H., WinfRed J.S.R.V., MidDELMANN T., Vogt S., WeYers S., LiPPHARdT B., Grosche G., Riehle F., SterR U. et Lisdat C. "The ${ }^{87} \mathrm{Sr}$ optical frequency standard at PTB". Metrologia, 48(5), 2011, 399.

[39] Hong F.L., Musha M., TaKamoto M., Inaba H., YANAGIMACHI S., TAKAMIZAWA A., WATABE K., IKEgAMI T., IMAE M., FUJII Y., AMEMIYA M., NAKAGAWA K., UEDA K. et KaTORI H. "Measuring the frequency of a Sr optical lattice clock using a $120 \mathrm{~km}$ coherent optical transfer". Opt. Lett., 34(5), 2009, 692-694.

[40] Yamaguchi A., Shiga N., Nagano S., Li Y., IshiJima H., Hachisu H., KumagaI M. et IDo T. "Stability Transfer between Two Clock Lasers Operating at Different Wavelengths for Absolute Frequency Measurement of Clock Transition in ${ }^{87} \mathrm{Sr}$ ". Appl. Phys. Express, 5(2), 2012, 022701.

[41] Matsubara K., Hachisu H., Li Y., Nagano S., Locke C., Nogami A., Kajita M., Hayasaka K., IDo T. et HosoKawA M. "Direct comparison of a $\mathrm{Ca}^{+}$singleion clock against a $\mathrm{Sr}$ lattice clock to verify the absolute frequency measurement”. Opt. Express, 20(20), 2012, 22034-22041.

[42] Hachisu H. et Ido T. "Intermittent optical frequency measurements to reduce the dead time uncertainty of frequency link". Jpn J. Appl. Phys., 54(11), 2015, 112401.

[43] Akamatsu D., Inaba H., Hosaka K., Yasuda M., OnAe A., Suzuyama T., AmEMiYa M. et Hong F.L. "Spectroscopy and frequency measurement of the ${ }^{87} \mathrm{Sr}$ clock transition by laser linewidth transfer using an optical frequency comb". Appl. Phys. Express, 7(1), 2014, 012401.

Article reçu le 5 janvier 2016, version révisée reçue le 8 juin 2016.
[44] Tanabe T., AKamatsu D., Kobayashi T., TAKAMIZAWA A., YANAGIMACHI S., IKEgAMI T., SuzuYama T., InABa H., OKUbo S., Yasuda M., HONG F.L., ONAE A. et HosaKa K. "Improved Frequency Measurement of the ${ }^{1} S_{0}-{ }^{3} P_{0}$ Clock Transition in ${ }^{87} \mathrm{Sr}$ Using a Cs Fountain Clock as a Transfer Oscillator". $J$. Phys. Soc. Jpn, 84(11), 2015, 115002.

[45] Yi-Ge L., Qiang W., Ye L., Fei M., BaI-Ke L., ERJun Z., Zhen S., FANG F., TIAN-ChU L. et ZhaN-JUN F. "First Evaluation and Frequency Measurement of the Strontium Optical Lattice Clock at NIM". Chinese Phys. Lett., 32(9), 2015, 090601.

[46] Laurent P., Abgrall M., Moric I., Lemonde P., Santarelli G., Clairon A., Bize S., Rovera D., GuÉNA J., SALOMON C. et al.. "PHARAO: le premier étalon primaire de fréquence, à atomes froids, spatial". Revue Française de Métrologie, 34, 2014, 3-21.

[47] Delva P. et LODEWYCK. J. "Atomic clocks: new prospects in metrology and geodesy." Acta Futura, 7, 2013, 67-78.

[48] Bondarescu R., Bondarescu M., Hetényi G., BOSCHI L., JETZER P. et BALAKRISHNA J. "Geophysical applicability of atomic clocks: direct continental geoid mapping". Geophys. J. Int., 191(1), 2012, 78-82.

[49] Dzuba V.A. et Flambaum V.V. "Relativistic corrections to transition frequencies of $\operatorname{Ag} I$, Dy $I, \mathrm{Ho} I, \mathrm{Yb} I I$, $\mathrm{Yb} I I I, \mathrm{Au} I$, and $\mathrm{Hg} I I$ and search for variation of the fine-structure constant". Phys. Rev. A, 77, 2008, 012515.

[50] Guéna J., Abgrall M., Rovera D., Rosenbusch P., Tobar M.E., Laurent P., Clairon A. et Bize S. "Improved Tests of Local Position Invariance Using ${ }^{87} \mathrm{Rb}$ and ${ }^{133}$ Cs Fountains". Phys. Rev. Lett., 109, 2012, 080801.

[51] WebB J.K., King J.A., MurPhy M.T., Flambaum V.V., CARSWELL R.F. et BAINBRIDGE M.B. "Indications of a Spatial Variation of the Fine Structure Constant". Phys. Rev. Lett., 107, 2011, 191101.

[52] LODEWYck J., BILICKI S., BoOKJans E., ROBYR J.-L., Shi C., Vallet G., Le Targat R., Nicolodi D., LE Coq Y., Guéna J., Abgrall M., Rosenbusch P. et BIZE S., "Optical to microwave clock frequency ratios with a nearly continuous strontium optical lattice clock", Metrologia, 53, 4, 2016, 1123-1130.

[53] Kessler T., Hagemann C., Grebing C., Legero T., STERR U., RIEHLE F., MARTIN M.J., CHEN L. et Ye J. "A sub-40-mHz-linewidth laser based on a silicon singlecrystal optical cavity”. Nat. Photon., 6, 2012, 687-692.

[54] Cole G.D., Zhang W., Martin M.J., Ye J. et ASPELMEYER M. "Tenfold reduction of Brownian noise in high-reflectivity optical coatings". Nat. Photon., 7, 2013, 644-650.

[55] Leibrandt D.R., ThORPe M.J., CHOU C.W., ForTIER T.M., DidDAMs S.A. et RosenBand T. "Absolute and Relative Stability of an Optical Frequency Reference Based on Spectral Hole Burning in $\mathrm{Eu}^{3+}: \mathrm{Y}_{2} \mathrm{SiO}_{5}$ ". Phys. Rev. Lett., 111, 2013, 237402. 